\title{
Turbulent Flow Measurements of the Boundary Layer on a Decelerating Flat Plate
}

\author{
by Kazu-hiro Mori*, Member Takio Hotta*, Member
}

\begin{abstract}
Velocity profiles, turbulence intensities and Reynolds stresses are measured in the turbulent boundary layer on a flat plate which is moving at a constant speed and is decelerated at a constant rate. The influence of the decelerating rate on the velocity profiles and turbulence quantities is clarified by comparing with those for steady cases. It is shown that the turbulence intensities of the decelerating boundary layer are larger than those under the steady condition. A similarity parameter for decelerated flows is derived from a dimensional analysis. The velocity profiles and the Reynolds stress distribution under the same parameter become a single curve. The turbulence closure model by Cebeci is examined through the experimental data.
\end{abstract}

\section{Introduction}

The problem of resistance of vessels under an unsteady condition is important, but there are not so many reports which are related to the turbulent boundary layer flows under a constant deceleration. Hirano and Fukuda ${ }^{1)}$ measured the turbulent boundary layer flow on a decelerating flat plate by using a hot film. They obtained the velocity profiles and boundary layer properties. The results showed that velocity profiles and momentum thicknesses were larger compared with the steady ones, but the shape parameter was nearly same.

Mori and $\mathrm{Liu}^{2)}$ reported mean velocity profiles measured on accelerating and decelerating flat plates together with numerical results. Their measurements were carried out by a small propeller current meter with a diameter of $3 \mathrm{~mm}$, while numerical computations were made to solve the unsteady turbulent boundary-layer equation with Cebeci's 0 -eq. model ${ }^{3)}$. The calculated results still suggested to be improved especially in the turbulence closure model. Cebeci's model was originally introduced to the flows around periodic motions, so the application to the present unsteady flows may not be suitable.

The purpose of this paper is to measure the turbulence intensities and the Reynolds stress in the boundary layer on a decelerated flat plate and to investigate the effect of the deceleration on its flow characteristics. The relation between such turbulence quantities and the decelerating rate is examined. It is also tried to derive a similarity para-

* Faculty of Engineering, Hiroshima University meter which may be required for decelerated flows.

In the present experiments a hot-film anemometer is utilized to measure the turbulence of the boundary layer flow on a plate. The plate towed steadily is decelerated at a constant rate. Turbulence components $u$ and $v$ are measured, where $u$ is in the uniform flow direction and $v$ in the direction normal to the plate. Turbulence intensity and Reynolds stress are defined similarly to the definition under the steady condition. The intensities of $u$ and $v$ and Reynolds stress are obtained at several reference velocities. Turbulence quantities are acquired for the different decelerations and different measuring positions. The influence of the rate of deceleration on the turbulence intensities and Reynolds stress are discussed and Cebeci's model is examined through the measured data. A similarity parameter for the accelerated and decelerated flows is also discussed, which is derived from a dimensional analysis.

\section{Experiment}

\section{1 Equipment and measurements}

The present experiment was carried out in the towing tank at Hiroshima University. Turbulence measurements were made by using a hot-film anemometer of the constant temperature type. The sensor is $51 \mu \mathrm{m}$ in diameter and coated by quartz. The flat plate is towed at the initial speed of $2 \mathrm{~m} / \mathrm{s}$ in each run and then retarded at a constant deceleration rate to rest. The water temperature is 8. $0^{\circ} \mathrm{C}$ to $12.5^{\circ} \mathrm{C}$, so that Reynolds number $U_{0} L / \nu=$ $5.4 \times 10^{6}$ and $U_{e} X_{o} / \nu=0.69 \times 10^{6}$ to $4.15 \times 10^{6}$, where $U_{0}$ and $U_{e}$ are the initial speed of plate and the boundary layer edge velocity respectively and L, 


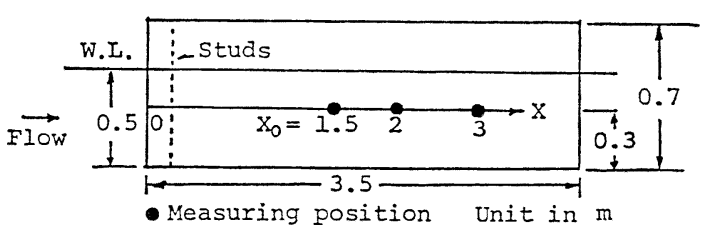

Fig. 1 Flat plate and measuring positions

Table 1 Position $X_{0}$ and deceleration $\alpha$

\begin{tabular}{|r|ccc|}
\hline$\alpha x$ & 1.5 & 2.0 & 3.0 \\
\hline$-0.01 \mathrm{~g}$ & & & $\Delta$ \\
\hline$-0.02 \mathrm{~g}$ & $\Delta$ & $\square$ & 0 \\
\hline$-0.03 \mathrm{~g}$ & & 0 & \\
\hline$-0.04 \mathrm{~g}$ & 0 & & \\
\hline
\end{tabular}

$X_{0}$ are the plate length and the distance along the plate from the leading edge to the measuring positions, respectively. Turbulence stimulators are used to assure the boundary layer flow to be turbulent in every case.

The used flat plate is $3.5 \mathrm{~m}$ long, $0.7 \mathrm{~m}$ wide and $5 \mathrm{~mm}$ thick whose leading edge is tapered to have a knife edge. Its draft is $0.5 \mathrm{~m}$ and turbulence stimulating studs are planted, at the position of $5 \%$ plate length from the leading edge, as shown in Fig. 1. Coordinates $x$ and $y$ are taken in the flow direction and normal to the plate surface, respectively, whose origin is at the leading edge and on the plate. Measurements are carried out along the $y$ axis at three measuring positions of $X_{0}$ $=1.5,2.0$ and $3.0 \mathrm{~m}$ for the steady and unsteady conditions.

In the unsteady cases the plate velocity is retarded at constant deceleration rates $\alpha$. The deceleration $\alpha$ is varied from $-0.01 g$ to $-0.04 g$ depending on the measuring positions $X_{0}$, where $g$ denotes the acceleration of gravity, $9.8 \mathrm{~m} / \mathrm{s}^{2}$. The combinations of $\alpha$ and $X_{0}$ are selected by taking account of similarity as discussed later. For the steady cases, i. e., $\alpha=0$, measurements are carried out at the speeds of 0.8 and $1.6 \mathrm{~m} / \mathrm{s}$. The experimental conditions are tabulated in Table 1 . The hot-film probe is set to make the probe axis parallel to the plate and measured at one point while the plate is decelerated. Signals of velocities $U+u$ and $V+v$ and the carriage speed $U_{c}$ are recorded into a datarecorder.

The distance and the time needed to stop from the initial speed of $2.0 \mathrm{~m} / \mathrm{s}$ are about $20 \mathrm{~m}$ and 20 sec for $\alpha=-0.01 \mathrm{~g}$, while about $5 \mathrm{~m}$ and $5 \mathrm{sec}$ for $\alpha=-0.04 \mathrm{~g}$. Velocity data are acquired at each processing time unit, $\Delta t$, after the plate deceleration begins. Seven boundary-layer edge velocities $U_{e}$ are referred for analysis at every $0.2 \mathrm{~m} / \mathrm{s}$ from $1.8 \mathrm{~m} / \mathrm{s}$ to $0.6 \mathrm{~m} / \mathrm{s}$ which are called reference velocities denoted by $U_{e r}$ here. When the deceleration is large as $-0.03 \mathrm{~g}$ or $-0.04 \mathrm{~g}$, the period of data acquisition is so short that several runs are carried out repeatedly to get enough number of data.

Calibration of the hot-film sensor is made in reference to the carriage speed $U_{c}$. Since the calibration curve can not be linear exactly, the velocities $U_{a}$ and $U_{b}$ are numerically determined, where $U_{a}$ and $U_{b}$ are the effective velocities obtained from two sensors. The velocity components $U+u$ and $V+v$ are obtained in terms of $U_{a}$ and $U_{b}$ by the relations $U+u=\left(U_{a}+U_{b}\right) / 2, V+v=\left(U_{a}-U_{b}\right) / 2$, where $U$ and $u$ are the mean and fluctuating velocities in the probe axis direction, while $V$ and $v$ normal to the axis.

\subsection{Data processing}

Turbulence quantities are obtained by following procedure. In the steady case the mean velocity $U$ in the boundary layer is determined by an ensemble average of $(U+u)_{i}$, where the subscript $i$ means the data at the time $t_{i}$. The fluctuation component $u_{i}$ is obtained by subtraction of $U_{i}$ from ( $U$ $+u)_{i}$. Similarly $v_{i}$ is determined. The nondimensional turbulence intensities and the Reynolds stress are conventionally defined by

$$
\begin{aligned}
& \sqrt{\frac{\bar{u}^{2}}{U_{e}}}=\sqrt{\sum_{i}\left(\frac{u_{i}}{U_{e}}\right)^{2} / N}, \\
& \sqrt{\frac{\bar{v}^{2}}{U_{e}}}=\sqrt{\sum_{i}\left(\frac{v_{i}}{U_{e}}\right)^{2} / N}, \\
& \frac{-\overline{u v}}{U_{e}^{2}}=-\sum_{i} \frac{u_{i} v_{i}}{U_{e}^{2}} / N, \\
& i=1,2, \cdots, N .
\end{aligned}
$$

In the unsteady case, the quantities are evaluated with regard to $U_{e r}$, see Fig. 2 . First the time $t_{r}$ when the boundary layer edge velocity becomes $U_{e r}$ is determined by analyzing the carriage speed. The data between $t_{r}-1 / 2 \Delta t$ and $t_{r}+1 / 2 \Delta t$ are assumed as data to be processed. Secondly a linear curve fitting is made for the data between $t_{r} \pm 1 / 2$

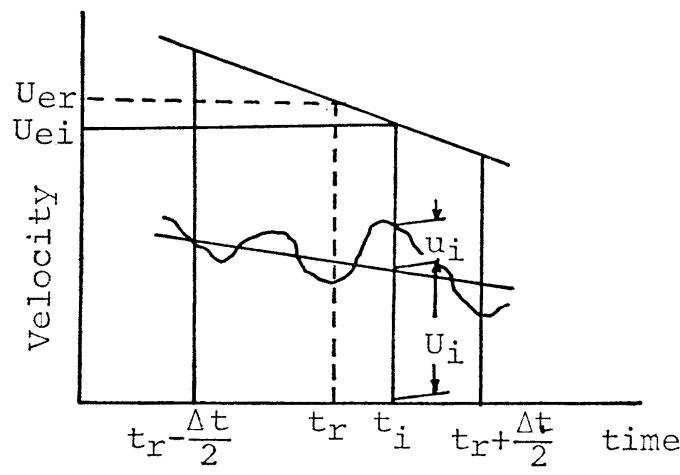

Fig. 2 Boundary layer velocity, $U_{e i}$ : boundary layer edge velocity, $U_{e r}$ : reference velocity, $U_{i}$ : average, $u_{i}$ : fluctuation, $\Delta t:$ processing time. 
$\Delta t$ by the least square method to determine $U_{i}$ at the time $t_{i}$. Then the fluctuation velocity $u_{i}$ is determined by $u_{i}=(U+u)_{i}-U_{i}$. The $v_{i}$ is determined by the same procedure.

In the present analysis $\Delta t$ is chosen as about $1 / 10$ of the time from the beginning of deceleration to the rest. The turbulence intensities and Reynolds stress are defined in a similar way to the steady case by using the ratios $u_{i} / U_{e i}$ and $v_{i} / U_{e i}$ as followings, where $U_{e i}$ is the boundary-layer edge velocity at $t_{i}$ and the ensemble average is denoted by a sym. bol .

$$
\begin{aligned}
& \sqrt{\left(\frac{u}{U_{e}}\right)^{2}}=\sqrt{\sum_{i}\left(\frac{u_{i}}{U_{e i}}\right)^{2} / N}, \\
& \sqrt{\left(\frac{v}{U_{e}}\right)^{2}}=\sqrt{\sum_{i}\left(\frac{v_{i}}{U_{e i}}\right)^{2} / N}, \\
& -\left(\frac{u v}{U_{e}^{2}}\right)=-\sum_{i}\left(\frac{u_{i} v_{i}}{U_{e i}{ }^{2}}\right) / N, \\
& i=1,2, \cdots, N .
\end{aligned}
$$

Here $N$ is the number of the sampled data. The sampling interval for $A-D$ conversion is $3 \mathrm{~m}$ sec. During a run, 666 data are sampled for one proces- sing time period in the case of $\alpha=-0.01 \mathrm{~g}$, and 333, $\alpha=-0.04 \mathrm{~g}$. Such numbers of data are not enough to determine reliably the turbulence quantities. The repetitions in the run are carried out for $N$ to be 1200 to 2200 .

\section{Results and discussion}

\section{1 Velocity profiles}

The velocity profiles in the boundary layer are shown in Fig. 3, in which the velocity $U$ is plotted against $y$. Figure a) shows the results at $X_{0}=1.5$ $\mathrm{m}, \alpha=-0.02 \mathrm{~g}$ and $-0.04 \mathrm{~g}$ and in b) $X_{0}=2.0 \mathrm{~m}$, $\alpha=-0.02 \mathrm{~g}$ and $-0.03 \mathrm{~g}$. The seven profiles for each deceleration are shown for every $0.2 \mathrm{~m} / \mathrm{s}$ of the outeredge velocities $U_{e}$ from $1.8 \mathrm{~m} / \mathrm{s}$ to 0.6 $\mathrm{m} / \mathrm{s}$. Every profile represents an inflection point in the range up to $y=20 \mathrm{~mm}$, i. e., the profile does not reduce monotonously with approaching the surface differing from the steady case. It is considered that such a velocity distribution is caused by the reduction of the fluid momentum induced by the backward shear stress transferred from the surface through deceleration. The same trend in profiles has been pointed out in the previous experiments ${ }^{12)}$, which is called the second boundary
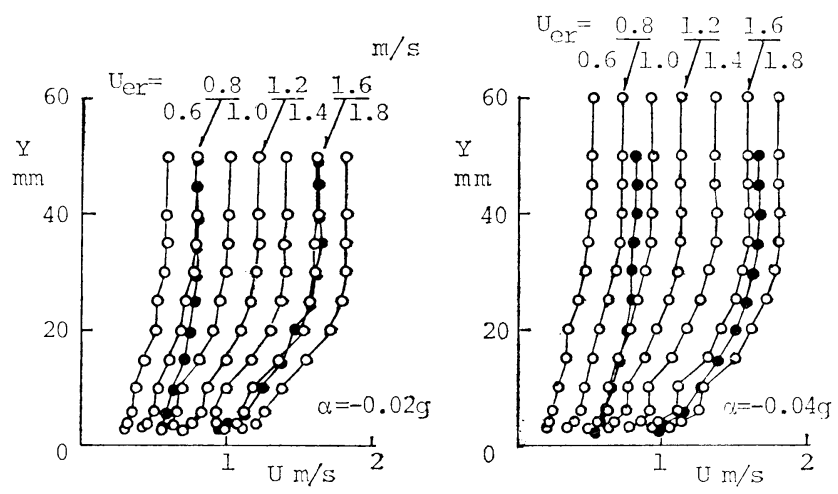

a) $\mathrm{XO}=1.5 \mathrm{~m}$

$$
\bullet: x=0.0
$$
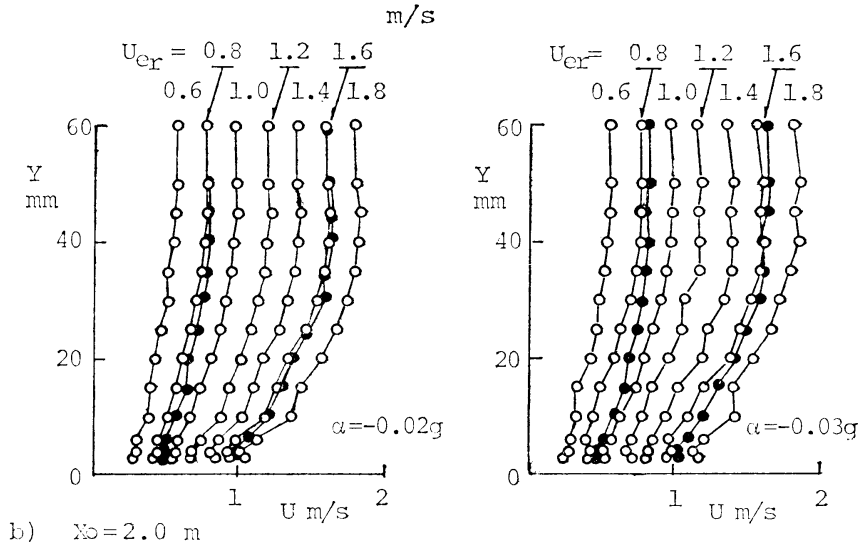

Fig. 3 Velocity profiles in the boundary layer on a decelerating flat plate, $U_{0} L / \nu=5.4 \times 10^{6}$ 
layer.

Displacement thickness $\delta^{*}$ and momentum thickness $\theta$ at $X_{0}=1.5 \mathrm{~m}$ and $2.0 \mathrm{~m}$ are shown in Fig. 4 together with the steady case. Both the thicknesses increase considerably with the reduction of the velocity $U_{e r}$ and those under the larger deceleration rate are thicker than those under smaller rate. The results at $X_{0}=1.5 \mathrm{~m}$ show much difference, i. e., about 1.5 times, between those of two decelerations of $-0.02 \mathrm{~g}$ and $-0.04 \mathrm{~g}$. The values of $\delta^{*}$ and $\theta$ in the steady case have almost the same values as those for the decelerating cases in the early stage, but show apparently thinner values at $U_{e r}=$ $0.8 \mathrm{~m} / \mathrm{s}$ compared with the deceleration cases.

\subsection{Turbulence intensities and Reynolds} stress

Nondimensional turbulence intensities $\sqrt{\left(\frac{\tilde{u}}{U_{e}}\right)^{2}}$, $\sqrt{\left(\frac{v}{U_{e}}\right)^{2}}$ and Reynolds stress rate $-\left(\widetilde{\frac{u v}{U_{e}^{2}}}\right)$ at $X_{0}$ $=1.5 \mathrm{~m}$ are shown in Fig. 5 for three deceleration rates of $\alpha=-0.04 g-0.02 g$ and 0.0 , i. e., steady case. They are plotted for seven reference velocities $U_{e r}$ covering from $1.8 \mathrm{~m} / \mathrm{s}$ to $0.6 \mathrm{~m} / \mathrm{s}$. The unsteady results are giving reasonable distributions, which assures that the descriptions of the turbulence intensity and Reynolds stress by the present ensemble procedure are reasonable for unsteady turbulent flows also.

In the $u$-intensity, there are peak values near the wall in the case of $U_{e r}=1.8 \mathrm{~m} / \mathrm{s}$. With the reduction of $U_{e r}$, the peak values become not so sharp, and the intensity distribution expands towards the outer region of the layer. Such an expansion is more dominant for larger deceleration, $\alpha=-0.04 \mathrm{~g}$. This is because of that the boundary layer thickness of $\alpha=-0.04 \mathrm{~g}$ is appreciably thicker in comparison with that of $\alpha=-0.02 \mathrm{~g}$, as shown in Fig. $3 \mathrm{a}$ ). The $u$-intensities in the deceleration case are higher than those in the steady case at $U_{e r}=$ $0.8 \mathrm{~m} / \mathrm{s}$. Such higher intensities can be understood from the fact that the velocity gradient $d U / d y$ under the deceleration is higher than that for the steady case. The $v$-intensities are about one half the $u$-intensities in every reference velocity, but the profiles have the same trend as the $u$-intensity distributions.

The Reynolds stress distributions, shown in the third row of Fig. 5, have peak values near the wall which shift away from the wall with the reduction of $U_{e r}$. The values for the steady case, at $U_{e r}=$ 1.6 and $0.8 \mathrm{~m} / \mathrm{s}$, have higher peaks with approaching the wall, while in the deceleration the peaks are not so sharp and away from the wall. It may be deduced that the Reynolds stress close to the wall in the deceleration is smaller than that in the steady case.

\section{3 The influence of $x_{0}$ and $\alpha$ on the turbu- lence quantities.}

It is inspected how the turbulence intensities and Reynolds stress are affected by the longitudinal distance of $X_{0}$ and the deceleration $\alpha$. Fig. 6 shows the $u$ - and $v$-intensities and Reynolds stresses at $X_{0}=1.5,2.0$ and $3.0 \mathrm{~m}$. The distributions of $u$ intensities show peaks near the wall for all positions. The amount of intensity becomes larger according to the increases of $X_{0}$ and the boundary layer thickness. Both the values of $u$ - and $v$-intensities in the deceleration are larger than those in the steady case. At $X_{0}=1.5 \mathrm{~m}$, the turbulence intensities of the larger deceleration $\alpha=-0.04 \mathrm{~g}$ show larger values, while at $X_{0}=3.0 \mathrm{~m}$ those of smaller deceleration seem to be also larger At
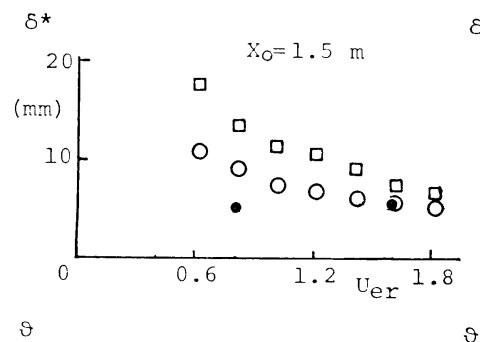

$\vartheta$

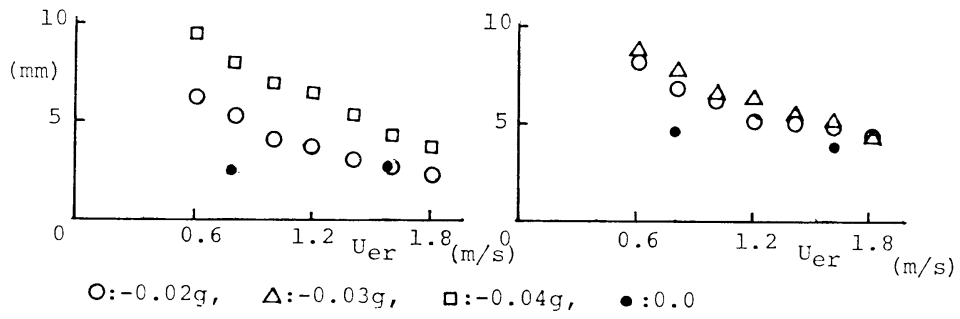

Fig. 4 Displacement thickness $\delta^{*}$ and momentum thickness $\vartheta$ against reference velocity $U_{e r}, \quad \mathrm{U}_{o} L / \nu=4.5 \times 10^{6}$ 

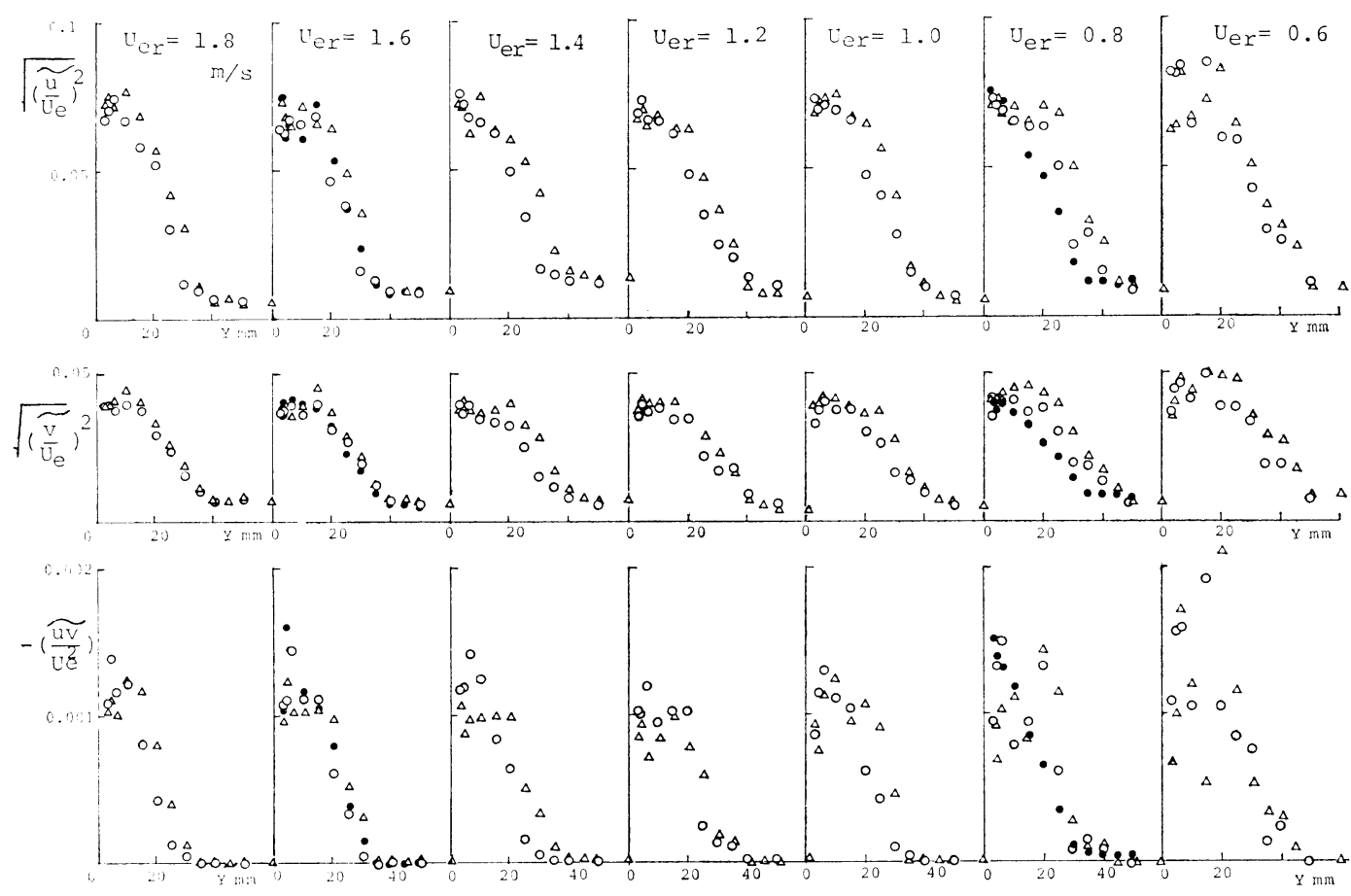

$\Delta ; a=-0.04 \mathrm{~g}, \quad 0 ; a=-0.02 \mathrm{~g}, \quad \bullet ; a=0.0$

Fig. 5 Turbulence intensities and Reynolds stress, $X_{0}=1.5 \mathrm{~m}, U_{0} L / \nu=5.4 \times 10^{6}$

$X_{0}=1.5 \mathrm{~m}$ the Reynolds stress of the larger deceleration $\alpha=-0.04 \mathrm{~g}$ is somewhat larger, while at $X_{0}=3.0 \mathrm{~m}$ those of the smaller deceleration $\alpha=$ $-0.01 \mathrm{~g}$ give larger values. This may be suggesting an existence of close relation between $\alpha$ and $X_{0}$.

\section{4 Similarity law for decelerated flow}

Introducing nondimensional parameters such as

$$
\begin{aligned}
U^{\prime} & =\frac{U}{U_{0}}, \quad V^{\prime}=\frac{V}{U_{0}} \sqrt{R_{x}}, \\
u^{\prime} & =\frac{u}{U_{0}}, \quad v^{\prime}=\frac{v}{U_{0}} \sqrt{R_{x}}, \\
x^{\prime} & =\frac{x}{X_{0}}, \quad y^{\prime}=\frac{y}{X_{0}} \sqrt{R_{x}}, \quad R_{x}=U_{0} X_{0} / \nu, \\
t^{\prime} & =\frac{t}{U_{0}}, \quad U_{e}^{\prime}=\frac{U_{e}}{U_{0}},
\end{aligned}
$$

the boundary layer equation is given by

$$
\begin{aligned}
& \frac{\alpha X_{0}}{U_{0}^{2}}\left(\frac{\partial U}{\partial t}-\frac{\partial U_{e}}{\partial t}\right)+U \frac{\partial U}{\partial x}+V \frac{\partial U}{\partial y} \\
& =\frac{\partial^{2} U}{\partial y^{2}}+\frac{\partial}{\partial y}(-\overline{u v}),
\end{aligned}
$$

where the primes are omitted. Eq. (4) suggests that the motion can be the same if the nondimensional parameter $\alpha X_{0} / U_{0}{ }^{2}$ is the same between different flows; $\alpha X_{0} / U_{0}^{2}$ is the second parameter for the decelerated flow. In other words, if the two flows have the same values of $R_{x}$ and $\alpha X_{0} / U_{0}{ }^{2}$, the mean velocity profiles and the Reynolds stress which are appearing in Eq. (4) may give the same profiles. Fig. 7 shows the velocity profiles for $\alpha X_{0} / U_{0}^{2}=-0.0735$ and -0.147 . Although the measuring positions and the deceleration rates are different, they are giving almost a single curve for each value of $\alpha X_{0} / U_{0}^{2}$. Fig. 8 shows the nondimensional Reynolds-stress distributions which are not so single as the mean velocity shown in Fig. 7. This may be partially due to the scattering of the original data.

\subsection{Discussion on Cebeci's closure model}

The Reynolds stress $-\rho \overline{u v}$ is commonly expressed in terms of the eddy viscosity coefficient $\rho \varepsilon_{m}$ as $-\rho \overline{u v}=\rho \varepsilon_{m} \partial U / \partial y$. Cebeci gives a 0 -equation model for $\varepsilon_{m}$ of an unsteady boundary layer flow ; $\boldsymbol{\varepsilon}_{m}$ is given by $\boldsymbol{\varepsilon}_{m i}$ and $\boldsymbol{\varepsilon}_{m_{0}}$ for the inner and outer regions, respectively.

$$
\begin{aligned}
& \varepsilon_{m i}=0.16 y^{2}\{1-\exp (-y / A)\}^{2}\left|\frac{\partial U}{\partial y}\right| \\
& \varepsilon_{m 0}=\beta \int_{0}^{\infty}\left(U_{e}-U\right) d y,
\end{aligned}
$$

where

$$
\begin{aligned}
& A=26 \nu u_{\tau}^{-1}\left[1-11.8\left(P_{t}^{+}+P_{x}^{+}\right]^{-1 / 2}\right. \\
& u_{\tau}=\left(\frac{\tau_{w}}{\rho}\right)^{1 / 2}, P_{t}^{+}=\frac{\nu}{u_{\tau}{ }^{3}} \frac{\partial U_{e}}{\partial t}, \\
& P_{x}{ }^{+}=\frac{\nu U_{e}}{u_{\tau}{ }^{3}} \frac{\partial U_{e}}{\partial x} \\
& \beta=0.0168 \times 1.55 /(1+\pi) \\
& \pi=0.55\left[1-\exp \left(-0.243 z^{1 / 2}-0.293 z\right]\right. \\
& z=R_{\theta} / 425-1, R_{\theta}=U_{e} \theta / \nu \\
& \theta: \text { momentum thickness. }
\end{aligned}
$$



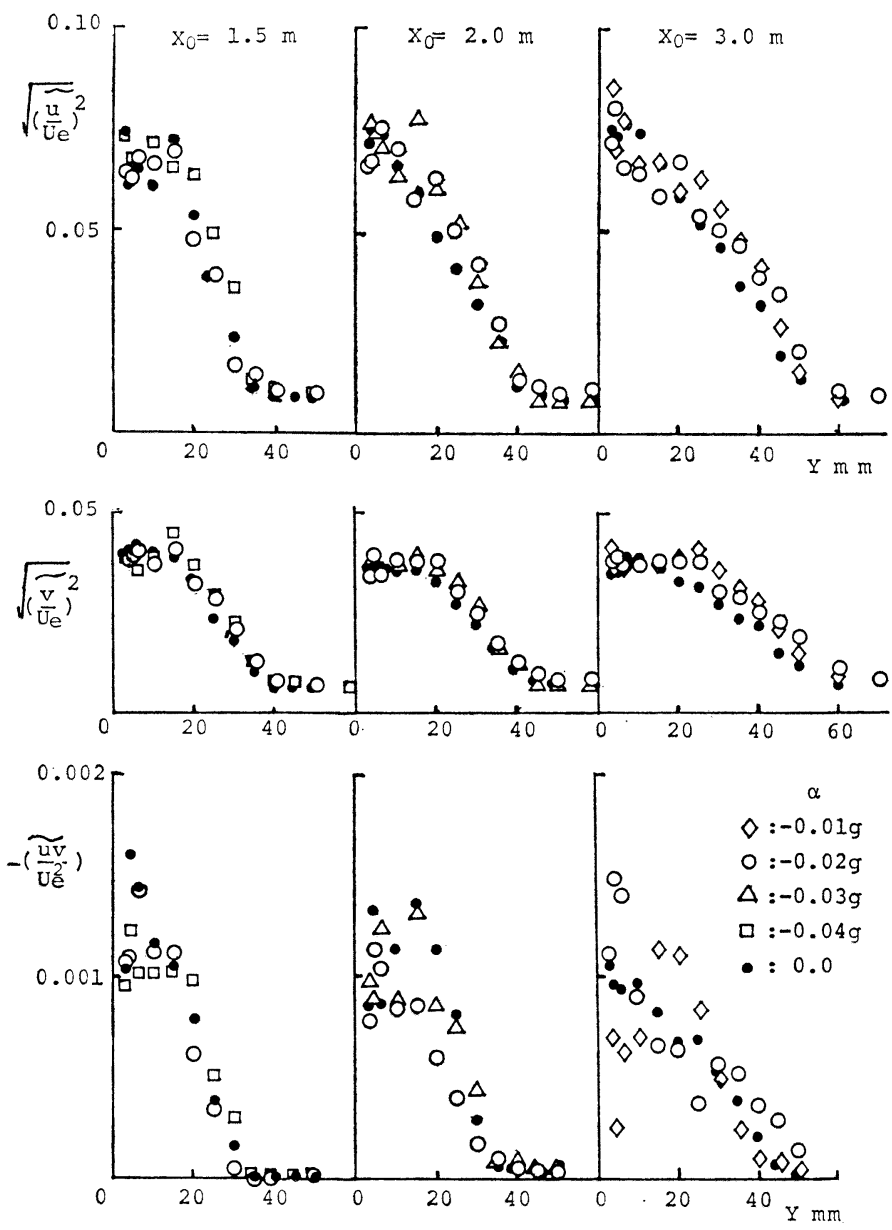

Fig. 6 Turbulence intensities and Reynolds stress at different measuring positions $X_{o}, U_{e r}=1.6 \mathrm{~m} / \mathrm{s}, \quad U_{o} L / \nu=5.4 \times 10^{6}$

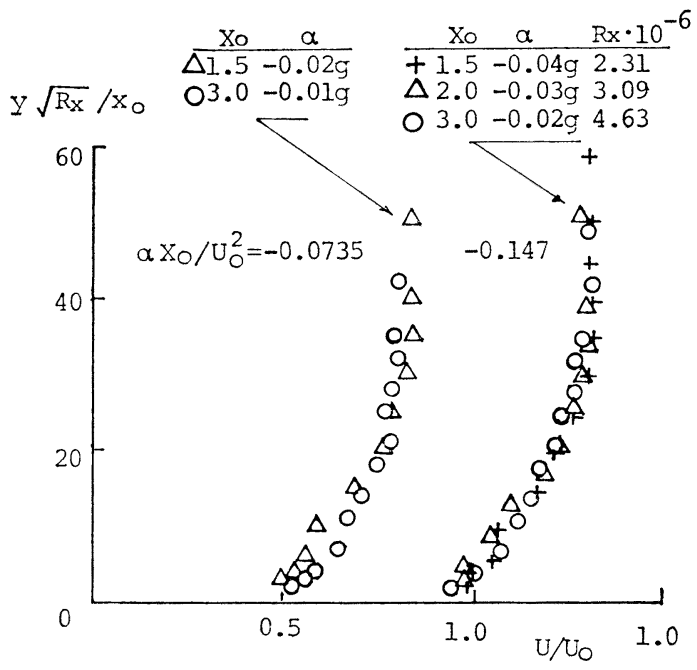

Fig. 7 Similarity of velocity profiles in the unsteady boundary layer for $U_{e r}=1.6$ $\mathrm{m} / \mathrm{s}, \quad R_{x}=U_{0} X_{o} / \nu$
In order to verify the model, which was invoked in the calculations ${ }^{21}$, the Reynolds stress derived by Cebeci's model is compared with the measured one. The results for the two cases at $U_{e r}=1.6$ and $0.8 \mathrm{~m} / \mathrm{s}$ are shown in Fig. 9 , where $X_{0}=1.5 \mathrm{~m}$ and $\alpha=-0.04 \mathrm{~g}$. Here the measured data, such as the velocity gradient, the displacement thickness and so on, are used in Cebeci's model. The skin friction $\tau_{w}$ is derived by the Ludwieg-Tillmann formula.

For the steady case, Cebeci's formula shows slightly larger values, but provides a good model to represent the Reynolds stress. For the unsteady case, however, the formula gives much larger stress than those directly measured. This tendency is more noticeable for $U_{e r}=0.8 \mathrm{~m} / \mathrm{s}$ than for $U_{e r}=$ $1.6 \mathrm{~m} / \mathrm{s}$.

As seen in Fig. 4, the displacement thickness at $U_{e r}=0.8 \mathrm{~m} / \mathrm{s}$ is much thicker than that at $U_{e r}=$ $1.6 \mathrm{~m} / \mathrm{s}$ and the inflection in the velocity profiles is more prominent for $U_{e r}=0.8 \mathrm{~m} / \mathrm{s}$. Such partic- 

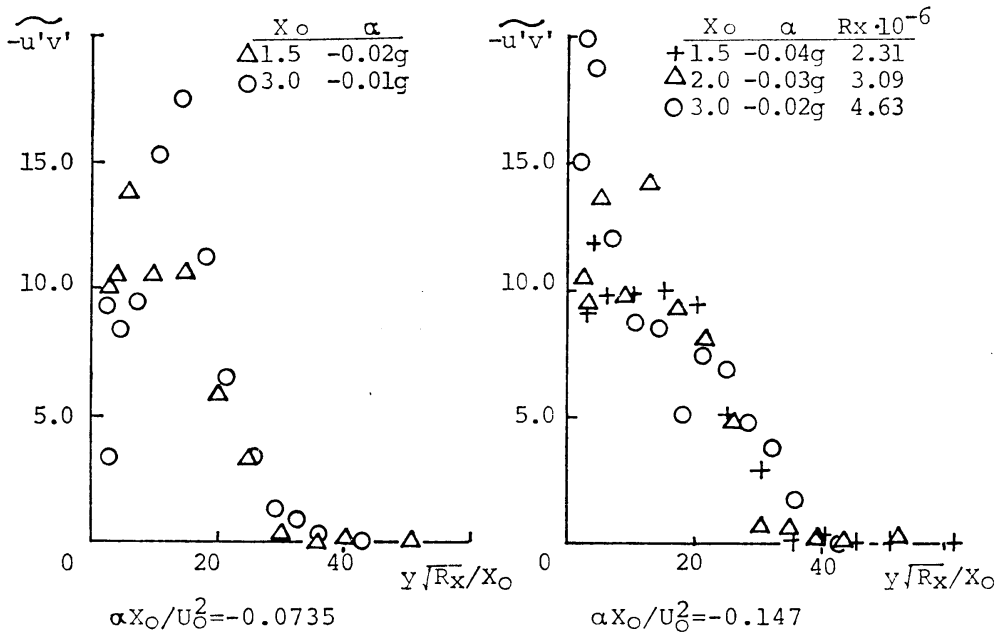

Fig. 8 Similarity of Reynolds stress distributions in the unsteady boundary layer for $U_{e r}=1.6 \mathrm{~m} / \mathrm{s}, \quad u^{\prime}=u / U_{o}, \quad v^{\prime}=v \sqrt{ } R_{x} / U_{o}, \quad R_{x}=U_{o} X_{o} / \nu$
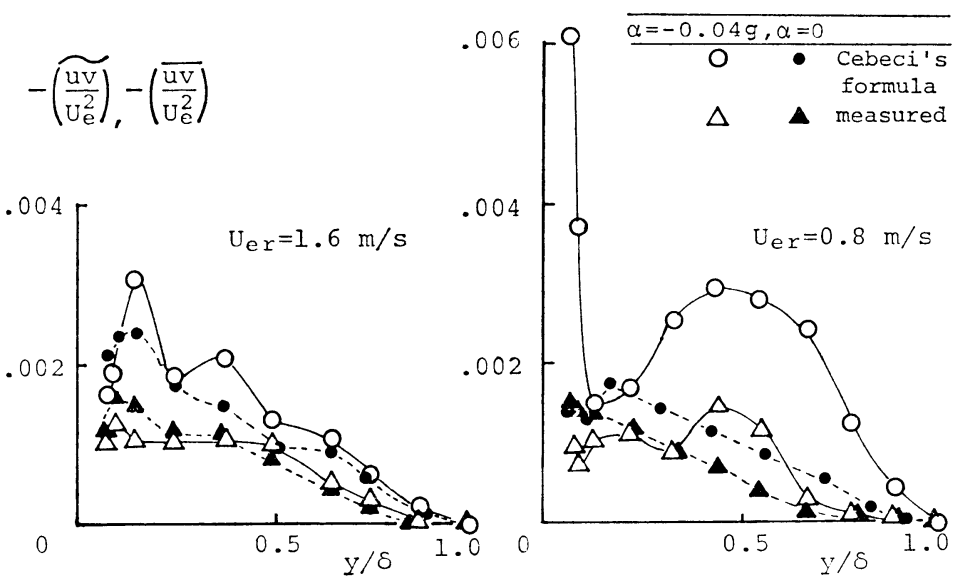

Fig. 9 Reynolds stresses by Cebeci's formula and measurements, at $X_{o}=1.5 \mathrm{~m}, U_{0} X_{0} / \nu=2.3 \times 10^{6}$

ularities due to the deceleration are not fully adopted in Cebeci's formula, so that the effect of the deceleration is exaggerated in the Reynolds stress.

In the boundary layer equation, the Reynolds stress appears only in terms of $\partial(-\overline{u v}) / \partial y$. In this connection the deviation in the gradient of the Reynolds stress is primarily important, which is observed close to the wall of the plate. This difference can be mentioned as one of the main reasons for the disagreements between the calculations and the measurements.

\section{Conclusion}

The turbulence measurements in the boundary layer on a decelerating flat plate are carried out and main conclusions obtained are summarized as follows ;
1) The turbulence quantities can be evaluated by an ensemble average procedure for the unsteady case as well as the quantities for the steady case.

2) The velocity profile under deceleration has an inflection point which is related to the development of the secondary boundary layer due to the deceleration.

3) The displacement and momentum thicknesses increase with the deceleration rate.

4) The turbulence intensities and the Reynolds stress of the unsteady boundary layer can be defined by the similar expression to those in the steady case.

5) The turbulence intensities of the boundary layer under deceleration are larger than those under steady condition.

6) A nondimensional parameter of $\alpha X_{0} / U_{0}^{2}$ can be a similarity parameter for the decelerated flow 
together with the Reynolds number.

7) Cebeci's expression for the eddy viscosity underestimates the effect of the deceleration and gives a larger value.

\section{Acknowledgment}

The authors wish to express their gratitudes to Mr. S. Majima, Mr. K. Nishimoto and Mr. K. Nishio for their assistances in carrying out the experiments and the data processing as their graduate theses.

\section{References}

1) Hirano, S. and Fukuda, K.: Viscous Drag during Deceleration (1 st Report)-On a Flat
Plate during Uniform Deceleration, Jour. Kansai Soc. Nav. Arch., Japan, vol. 156 (1975) (in Japanese).

2) Mori, K. and Liu, Y.: On Boundary Layer Flow and Frictional Resistance of a Uniformly Decelerating or Accelerating Flat Plate, Memoirs of Faculty of Engineering, Hiroshima Univ., vol.9, No. 2 (1986).

3) Cebeci, T.: Calculation of Unsteady TwoDimensional Laminar and Turbulent Bound. ary Layer with Fluctuations in External Velocity, Proc. of the Royal Soc. of London, Ser. A, vol. 355 (1977). 